\title{
Erratum to: Tumor Static Concentration Curves in Combination Therapy
}

\author{
Tim Cardilin, ${ }^{1,2,8}$ Joachim Almquist, ${ }^{1,3}$ Mats Jirstand, ${ }^{1}$ Alexandre Soselly, ${ }^{4,5}$ Christiane Amendt, ${ }^{6}$ \\ Samer El Bawab, ${ }^{4}$ and Johan Gabrielsson ${ }^{7}$
}

\section{Erratum to: AAPS J}

\section{DOI: $10.1208 / \mathbf{s} 12248-016-9991-1$}

Equation 6: The complete system should read

$\frac{d V_{1}}{d t}=I\left(\mathrm{C}_{\text {cetuximab }}\right) k_{g} V_{1}-S\left(\mathrm{C}_{\text {cisplatin }}\right) k_{k} V_{1}$

$\frac{d V_{2}}{d t}=S\left(\mathrm{C}_{\text {cisplatin }}\right) k_{k} V_{1}-k_{k} V_{2}$

$\frac{d V_{3}}{d t}=k_{k} V_{2}-k_{k} V_{3}$

$\frac{d V_{4}}{d t}=k_{k} V_{3}-k_{k} V_{4}$

Results: Drug exposure models

The parameter value for the cetuximab model should be $k_{e}=0.017 \mathrm{~h}^{-1}, k_{a}=0.44 \mathrm{~h}^{-1}, F=1$, and $V=94 \mathrm{~mL} \cdot \mathrm{kg}^{-1}$.

The parameter values for the cisplatin model should be $k_{a}=42 \mathrm{~h}^{-1}, k_{10}=1.3 \mathrm{~h}^{-1}, k_{12}=3.3 \mathrm{~h}^{-1}, k_{21}=0.047 \mathrm{~h}^{-1}, \quad F=1$, and $V_{p}=377 \mathrm{~mL} \cdot \mathrm{kg}^{-1}$.

Table I scribed by the following system of differential equations"

Equation 36: The vectors should be

$$
\begin{aligned}
\boldsymbol{w}_{2,2} & =(0,0, \ldots, 0,1,0), \boldsymbol{w}_{2,3}=(0, \ldots 0,1,0,0), \ldots, \boldsymbol{w}_{2, n} \\
& =(0,1,0, \ldots, 0)
\end{aligned}
$$

The online version of the original article can be found at http:Ildx.doi.org10.1208/s12248-016-9991-1.

\footnotetext{
${ }^{1}$ Fraunhofer-Chalmers Centre, Chalmers Science Park, Gothenburg, Sweden.

${ }^{2}$ Department of Mathematical Sciences, Chalmers University of Technology and University of Gothenburg, Gothenburg, Sweden.

${ }^{3}$ Department of Biology and Biological Engineering, Chalmers University of Technology, Gothenburg, Sweden.

${ }^{4}$ Global Early Development-Quantitative Pharmacology and Drug Disposition, Quantitative Pharmacology, Merck, Darmstadt, Germany.

${ }^{5}$ Present Address: Pharmaceutical Research and Early Development, Hoffmann-Le Roche, Basel, Switzerland.

${ }^{6}$ Translation Innovation Platform Oncology, Merck, Darmstadt, Germany.

${ }^{7}$ Department of Biomedical Sciences and Veterinary Public Health, Swedish University of Agricultural Sciences, Uppsala, Sweden.
} 